\title{
Discriminatory Dissolution Method Development and Validation of Etoricoxib Tablets
}

\author{
Yasvanth Ashokraj*, Atul Daroi, Raja Gupta, Aruna Khanolkar, \\ Amol Kulkarni, Swati Laud, Milind Pokale, Sanjeet Shedge, and Praveen Date \\ CIPLA Ltd, Mumbai, India
}

\section{ABSTRACT}

e-mail: yasvanth.ashokraj@cipla.com

The availability of a discriminatory dissolution method for use in either quality control testing or product evaluation in quality by design trials is mandatory for products intended for the developed market. This study demonstrates the systematic development of a discriminatory dissolution method for etoricoxib, a BCS Class 2 drug exhibiting highly pHdependent solubility. The $\mathrm{pH}$, speed, and apparatus were simultaneously optimized and tested for method variability, reproducibility, and robustness. The discriminative power of the developed method was suitably demonstrated.

KEYWORDS: Etoricoxib; dissolution; discriminatory; BCS Class 2.

\section{INTRODUCTION}

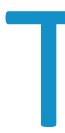

he intestinal absorption of drug from a dosage form after oral administration constitutes the following sequential processes: (1) release of the drug substance from the drug product; (2) dissolution, solubilization, or both of the drug; and (3) permeability of the dissolved drug at the site of absorption. Because the first two steps are critical in determining bioavailability, the development of an appropriate in vitro dissolution method is essential in the prediction of in vivo performance (1).

In general, dissolution tests are useful for (1) assessing the characteristics of the active pharmaceutical ingredient (API) such as the particle size and the crystal form; (2) guiding development of new formulations; (3) assisting in proper formulation selection (selection of excipients) and optimizing the manufacturing process (e.g., compression forces, equipment) during dosage form optimization; (4) assessing the batch-to-batch quality of a drug product; (5) enabling the comparison of batches obtained from different production sources; (6) comparing new or generic formulations with an existing product; (7) assessing the stability of the drug product, helping in establishment of shelf life; (8) ensuring product quality in the case of certain scale-up and post-approval changes (SUPAC) like manufacturing site changes, increase or decrease of batch size, and small quantitative changes in excipients; (9) providing a basis for achieving an in vitro-in vivo correlation (predicting product performance in vivo); and (10) minimizing the need for bioequivalence studies (biowaivers) (2).
In particular, dissolution studies are quite predictive of in vivo performance for insoluble or poorly soluble drugs, where absorption is (i.e., Biopharmaceutics Classification System [BCS], Class 2 drugs). Furthermore, a dissolution method with suitable discriminating power is preferred to indicate any possible changes in the quality of the product before in vivo performance is affected. The discriminatory power of the dissolution method is the ability of the method to detect changes in drug product performance, generally demonstrated by determining the effect of deliberate changes in the formulation or process on dissolution characteristics.

The systematic development of a dissolution method of a discriminatory nature is required much earlier than at full-fledged product development. This enables the formulator to adopt the right approach in finalizing the prototype formula and process for further scale-up. In addition, demonstration of the discriminatory nature of the dissolution method to be used in routine quality control of commercial lots is expected by major regulatory agencies worldwide $(3,4)$.

Etoricoxib (5-chloro-2-(6-methylpyridin-3-yl)-3- (4-methyl sulfonylphenyl)pyridine) is an orally active, selective cyclooxygenase-2 inhibitor (5). It is a widely prescribed anti-inflammatory drug available in tablet strengths of $30,60,90$, and $120 \mathrm{mg}$. It is a poorly soluble and highly permeable BCS Class 2 drug (6). The pKa of etoricoxib is 4.6, and the drug exhibits $\mathrm{pH}$-dependent solubility (i.e., high solubility in gastric media [low $\mathrm{pH}$ ] and decreasing solubility with increasing $\mathrm{pH}$ ). The presence of bile salts and lecithin in biorelevant media does not appear to 
impact its solubility (6). There is no quality control or discriminatory dissolution method for etoricoxib tablets reported in the literature. Therefore, the aim of the present work was to develop and validate a discriminatory dissolution method for etoricoxib tablets.

\section{MATERIALS AND METHODS}

All solvents used for HPLC analysis were of HPLC grade, and all other chemicals were of analytical reagent grade and were used as received. The following chemicals were used to prepare buffers and HPLC mobile phase: sodium perchlorate monohydrate (Merck, India), acetonitrile (Fisher Scientific, India), methanol (Fisher Scientific, India), sodium acetate trihydrate (Qualigens, India/Merck, India), glacial acetic acid (Fisher Scientific, India), potassium biphthalate (Merck, India), hydrochloric acid (sd Fine chemicals, India), potassium dihydrogen orthophosphate (sd Fine chemicals, India), sodium hydroxide pellets (Merck, India), and potassium chloride (Merck, India).

\section{Formulation Preparation}

Film-coated tablets of etoricoxib were prepared by two processes using the following excipients: calcium hydrogen phosphate anhydrous, croscarmellose sodium, magnesium stearate, microcrystalline cellulose, and Opadry.

\section{pH-Equilibrium Solubility Studies}

Equilibrium solubility at various $\mathrm{pHs}$ were determined by the shake-flask method. Etoricoxib was added to $25 \mathrm{~mL}$ of corresponding USP buffer solution $\mathrm{pH} \mathrm{1.2,} \mathrm{3.0,} \mathrm{4.5,} \mathrm{5.5,}$ and 6.8) until a heterogeneous system (solid sample and liquid) was achieved. The samples were sonicated for 10 min and placed in a shaker water bath maintained at $37^{\circ} \mathrm{C}$ after ensuring the presence of excess solid in the sample. The sample was stirred for $24 \mathrm{~h}$ to achieve equilibrium, a small quantity $(2 \mathrm{~mL})$ was sampled and filtered through a 0.45- $\mu \mathrm{m}$ PVDF filter, and the concentration of etoricoxib was determined by a validated HPLC method.

\section{Dissolution Studies}

All dissolution runs were performed on six tablets using USP Apparatus 2 with a medium volume of 900 $\mathrm{mL}$ maintained at $37{ }^{\circ} \mathrm{C}$ unless otherwise indicated. Dissolution profiles were generated by sampling at 5,10 , $15,20,30,45$, and $60 \mathrm{~min}$. Additionally, time points at 90 $\mathrm{min}$ and infinity (200 rpm for $10 \mathrm{~min}$ ) were also generated in some profiles. Samples were filtered through 0.45$\mu \mathrm{m}$ PVDF filters and analyzed for etoricoxib content by a validated HPLC method.

\section{Sample Analysis}

Sample analysis was performed using a Waters 2695 separation module equipped with a 2487 UV-vis detector (Waters Corp., Milford, MA, USA). The validated reversedphase HPLC method employed a Hypersil BDS C-18 column (10 $\mathrm{cm} \times 4.6 \mathrm{~mm}, 3-\mu \mathrm{m}$ particle size) maintained at $25^{\circ} \mathrm{C}$ with a mobile phase composed of $10 \mathrm{mM}$ sodium perchlorate monohydrate/acetonitrile (55:45) at a flow rate of $1.5 \mathrm{~mL} / \mathrm{min}$ with detection at $235 \mathrm{~nm}$.

\section{RESULTS AND DISCUSSION}

Major regulatory agencies $(3,4,7)$ expect discriminatory dissolution methods for the quality control of the pharmaceutical products, especially oral solid dosage forms. A report by Fish et al. (8) outlined a comprehensive dissolution method development strategy to achieve a discriminatory method that was elegantly showcased for dasatinib film-coated tablets. Accordingly, the following steps are required for any discriminatory dissolution method development: (1) set the objective to achieve a dissolution profile (e.g., $<50 \%$ in $15 \mathrm{~min}$ and $>85 \%$ in $30 \mathrm{~min}$ ); (2) design the experimental flowchart for the method development strategy with anticipated results and way-forward actions; (3) systematically optimize and finalize the medium, speed, apparatus, and volume parameters of the dissolution method; (4) demonstrate the discriminatory nature of the optimized dissolution method; (5) demonstrate reproducibility of results and robustness of the method, satisfying variability requirements (i.e., $<20 \%$ RSD at first sampling point and $<10 \%$ RSD at other sampling points). In addition, it is desirable to achieve sink condition requirements, where sink conditions occurs when the amount of drug that can be dissolved in the dissolution medium is three times greater than the amount of drug to be dissolved (2). However, deviation from fulfilling sink conditions is acceptable if the discriminatory nature of the dissolution method is proved satisfactorily (7).

Understanding the physicochemical characteristics of the API is a key starting point for any dissolution method development. In particular, information on equilibrium solubility provides quick direction on the selection of a suitable dissolution medium. The results of the equilibrium solubility studies for etoricoxib demonstrate that its solubility is highly $\mathrm{pH}$-dependent, which is consistent with earlier reports (6). A significant difference in solubility was observed in the $\mathrm{pH}$ region of 1.2-3.0 (i.e., $22.52 \mathrm{mg} / \mathrm{mL}$ at $\mathrm{pH} 1.2$ and $1.44 \mathrm{mg} / \mathrm{mL}$ at $\mathrm{pH}$ 3.0). Solubility decreased further and remained constant $(0.12 \mathrm{mg} / \mathrm{mL})$ above $\mathrm{pH}$ 3.0.

According to the USP (7), it is essential to have a sufficient number of time points to adequately characterize the ascending and plateau phases of dissolution curves in a 
dissolution method. This was considered the primary criterion for the selection of dissolution conditions during dissolution method development for etoricoxib tablets. The objective was to obtain a profile with $<50 \%$ drug release in $10 \mathrm{~min}$ and $>80 \%$ drug release in 30 min with reasonable variability at each sampling point. As observed from the solubility data, dissolution of etoricoxib can be driven predominantly by the $\mathrm{pH}$ of the medium. The effect of $\mathrm{pH}$ on the dissolution of etoricoxib tablets using Apparatus 2 at 50 rpm is shown in Figure 1 , which shows dissolution studies performed at $\mathrm{pH} 1.2$, 4.5 , and 6.8 covering high, mid, and low solubility regions. As anticipated from the results of saturation solubility studies, rapid and complete dissolution was observed at low $\mathrm{pH}$, whereas slow and incomplete dissolution was seen at higher $\mathrm{pH}$. However, in the case of $\mathrm{pH} \mathrm{4.5,}$ a distinct profile indicating the ascending and plateau phases of the dissolution curve was obtained.

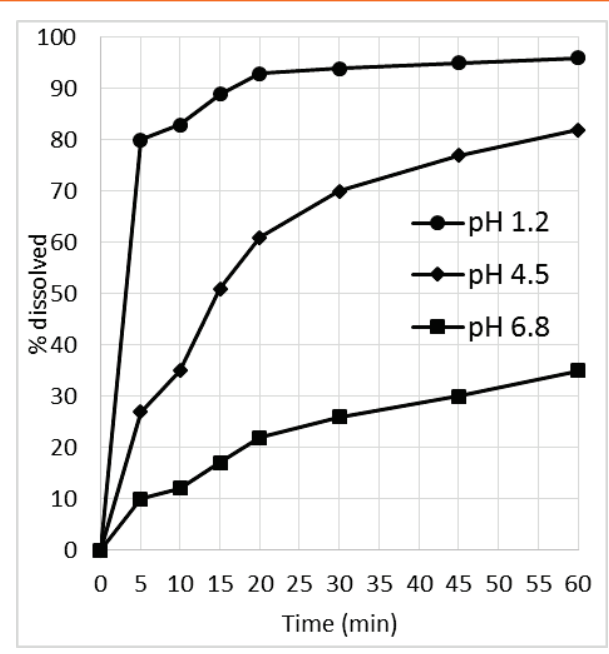

Figure 1. Dissolution profiles of etoricoxib tablets in different $\mathrm{pH}$ buffers. Dissolution conditions are $900 \mathrm{~mL}, 50 \mathrm{rpm}$, Apparatus 2.

Surfactants are generally utilized to improve solubility in terms of dissolution rate and extent. Since a significantly incomplete dissolution was observed in $\mathrm{pH} 6.8$, it appeared that a solubilizer (i.e., surfactant) would be necessary to achieve complete dissolution in $\mathrm{pH} 6.8$. However, Okumu et al. (6) demonstrated that addition of surfactant did not improve the solubility of etoricoxib by determining the solubility in blank fasted-state simulated intestinal fluid (FaSSIF), fed-state simulated intestinal fluid (FeSSIF), and FaSSIF/FeSSIF. Therefore, $\mathrm{pH}$ 4.5 without surfactant was selected to further evaluate the reproducibility and the discriminatory nature of the medium. Surprisingly, significant run-to-run variability was observed in $\mathrm{pH} 4.5$, which was not acceptable (data not shown). Thus, the method development effort was directed toward exploring variations in $\mathrm{pH}$ and speed, as given in Table 1, to achieve a balance between them that would provide a suitable dissolution method.

Table 1. Development Matrix for Dissolution Method of Etoricoxib Tablets

\begin{tabular}{|c|c|c|c|l|}
\hline \multirow{2}{*}{$\mathrm{pH}$} & \multicolumn{3}{|c|}{ rpm } & \multicolumn{1}{|l|}{ Comments } \\
\cline { 2 - 5 } & 50 & 75 & 100 & \\
\hline 1.2 & - & - & - & $\begin{array}{l}\text { Desired discrimination not anticipated due } \\
\text { to high solubility }\end{array}$ \\
\hline 2.0 & - & - & - & $\begin{array}{l}\text { Desired discrimination not anticipated due } \\
\text { to high solubility }\end{array}$ \\
\hline 3.0 & Y & FE & NA & $\begin{array}{l}\text { Desired discrimination anticipated at low } \\
\text { rpm, thus rest of the hydrodynamics was } \\
\text { reserved. }\end{array}$ \\
\hline 4.0 & Y & FE & NA & $\begin{array}{l}\text { Desired discrimination anticipated at low } \\
\text { rpm, thus rest of the hydrodynamics was } \\
\text { reserved. }\end{array}$ \\
\hline 4.5 & NA & Y & Y & Results in 50 rpm not reproducible. \\
\hline 5.0 & - & - & - & $\begin{array}{l}\text { Discarded due to low solubility. Evaluated in } \\
\text { pH 6.8. }\end{array}$ \\
\hline 6.0 & - & - & - & $\begin{array}{l}\text { Discarded due to low solubility. Evaluated in } \\
\text { pH 6.8. }\end{array}$ \\
\hline 6.8 & NA & Y & Y & $\begin{array}{l}\text { Desired discrimination not anticipated at } \\
\text { low rpm. }\end{array}$ \\
\hline $\begin{array}{l}\text { Y- experiments to be performed. } \\
\text { FE - follow-up experiments after reviewing the results. } \\
\text { NA - experiments not planned. }\end{array}$ \\
\hline
\end{tabular}

Dissolution conditions: Apparatus 2, $900 \mathrm{~mL}(n=6)$

Dissolution profiles of etoricoxib tablets evaluated with a combination of different $\mathrm{pH}$ and hydrodynamics using Apparatus 2 are shown in Figure 2. Drug dissolution did not meet the preset criterion at either 10 or $30 \mathrm{~min}$ in $\mathrm{pH}$ 3.0, 4.0, and 6.8 at 50, 75, or $100 \mathrm{rpm}$. This incomplete dissolution was due to either a slow dissolution rate related to the non-sink conditions of $\mathrm{pH} 6.8$ or insufficient hydrodynamics to break the diffusion layer for rapid mixing of dissolved drug in pH 3.0 and 4.0 (9). Dissolution profiles in pH 4.5 at $100 \mathrm{rpm}$ fulfilled the set objective, whereas at $75 \mathrm{rpm}$ the dissolution was still slow $(<80 \%$ in $30 \mathrm{~min}$ ). To understand the effect of apparatus at the given $\mathrm{pH}$, speed, and volume parameters, a dissolution profile was generated at $\mathrm{pH} 4.5$ in Apparatus 1. The results (Figure 3 ) show the non-suitability of Apparatus 1 at 100 rpm for etoricoxib tablets, which is attributed to the sticky mass left in the basket leading to uneven tablet dispersion and settling of dispersed particles at the bottom of the vessel. Therefore, the dissolution condition that was finalized after confirming the acceptable run-torun reproducibility (Figure 4) is $900 \mathrm{~mL}$ of pH 4.5 acetate buffer in Apparatus 2 at $100 \mathrm{rpm}$, though the impact of the volume parameter was not evaluated. 


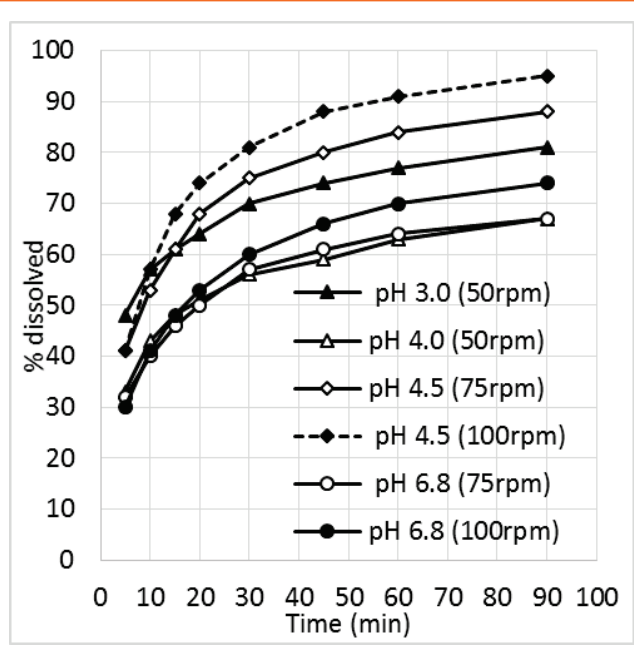

Figure 2. Dissolution profiles generated as per development matrix (Table 1).

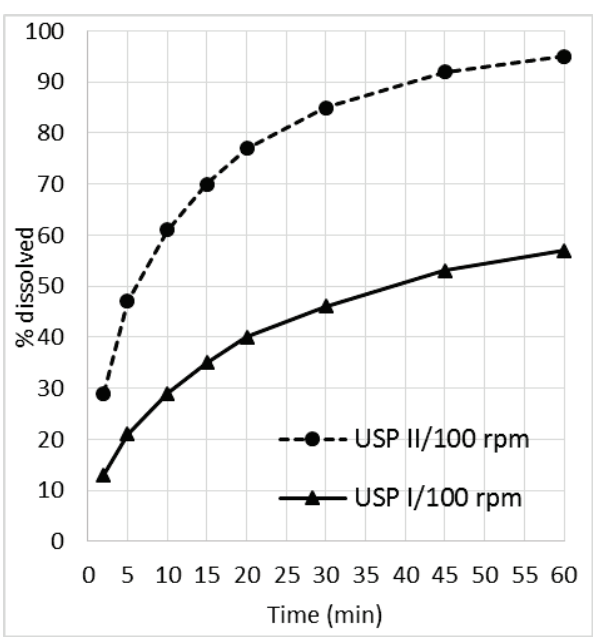

Figure 3. Effect of apparatus on dissolution of etoricoxib tablets in $900 \mathrm{~mL}$ of $\mathrm{pH} 4.5$ acetate buffer.

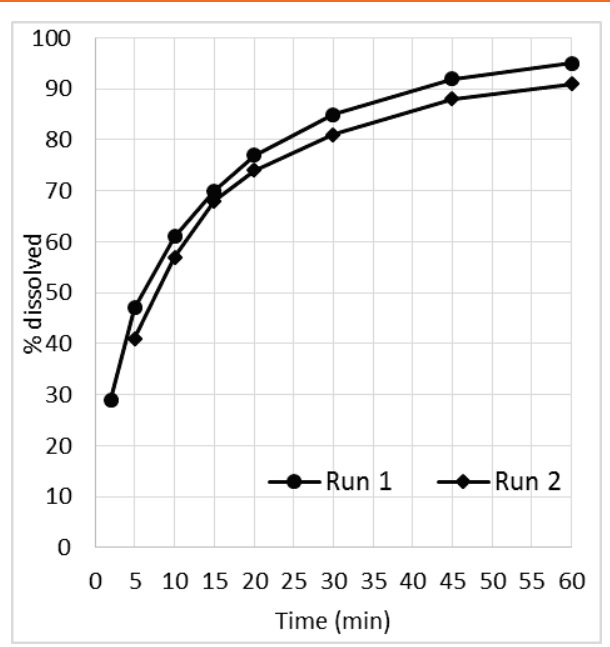

Figure 4. Dissolution profiles of the same lot of etoricoxib tablets to demonstrate the run-to-run reproducibility in $900 \mathrm{~mL} \mathrm{of} \mathrm{pH} 4.5$ buffer, 100 rpm, Apparatus 2.
A dissolution method that is capable of distinguishing significant changes in the composition and manufacturing process is defined as a discriminative method. The formulation utilized earlier for dissolution method development was manufactured by Process 1 using the excipients given above. Accordingly, two formulations considered significantly different, one with an increased calcium hydrogen phosphate anhydrous and the other manufactured by a different process (Process 2), were selected to demonstrate the discriminatory nature of the chosen dissolution method. Results are given in Figure 5. As anticipated, the dissolution rate of the formulation with increased calcium hydrogen phosphate anhydrous decreased significantly $\left(f_{2}\right.$ of 35.23$)$ compared with the standard formulation. This was attributed to the insoluble nature of calcium hydrogen phosphate anhydrous (10). On the other hand, the change in manufacturing process did not affect the dissolution rate significantly $\left(f_{2}\right.$ of $61.61)$, though a slightly slower profile was observed. This suitably demonstrates the discriminatory nature of the selected dissolution method.

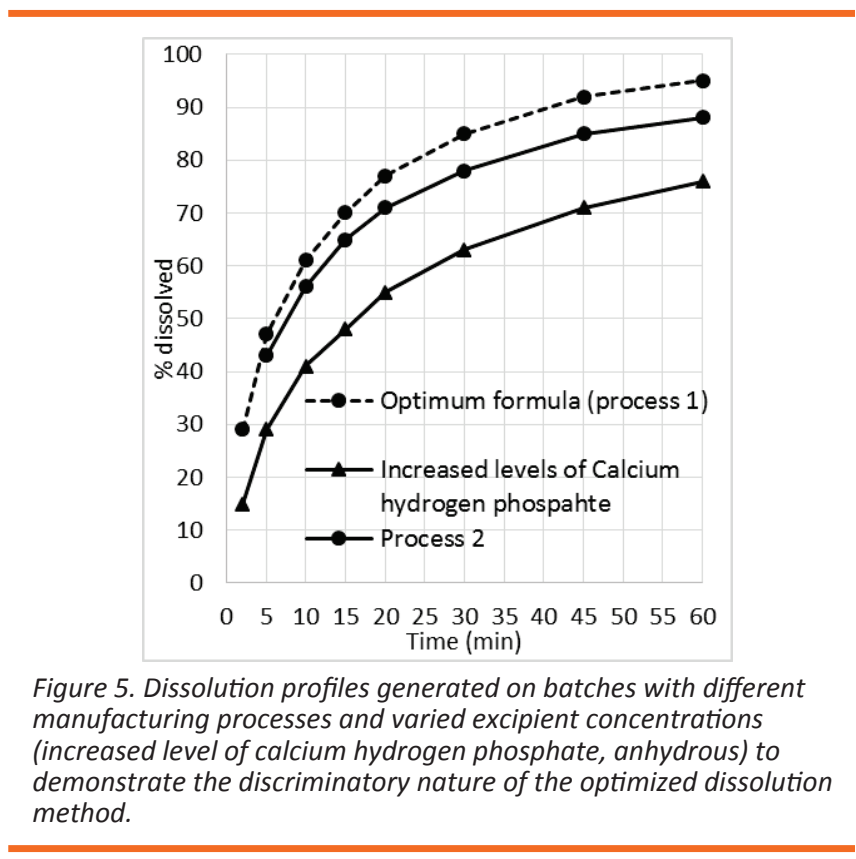

Further efforts were undertaken to assess the robustness of the method by careful examination of plausible parameters that could influence the results during routine analysis. Accordingly, changes in $\mathrm{pH}$ and grades of critical chemicals used in the preparation of buffer were evaluated. The $\mathrm{pH}$ of the medium was adjusted to $\pm 0.2 \mathrm{pH}$ units, and the source of sodium acetate was changed to study the impact on the dissolution of etoricoxib tablets. The resulting profiles (Figure 6A,B) almost overlap that of the standard formulation. Thus, the chosen dissolution 
method is robust enough to withstand slight changes in laboratory conditions to provide reproducible dissolution profiles.
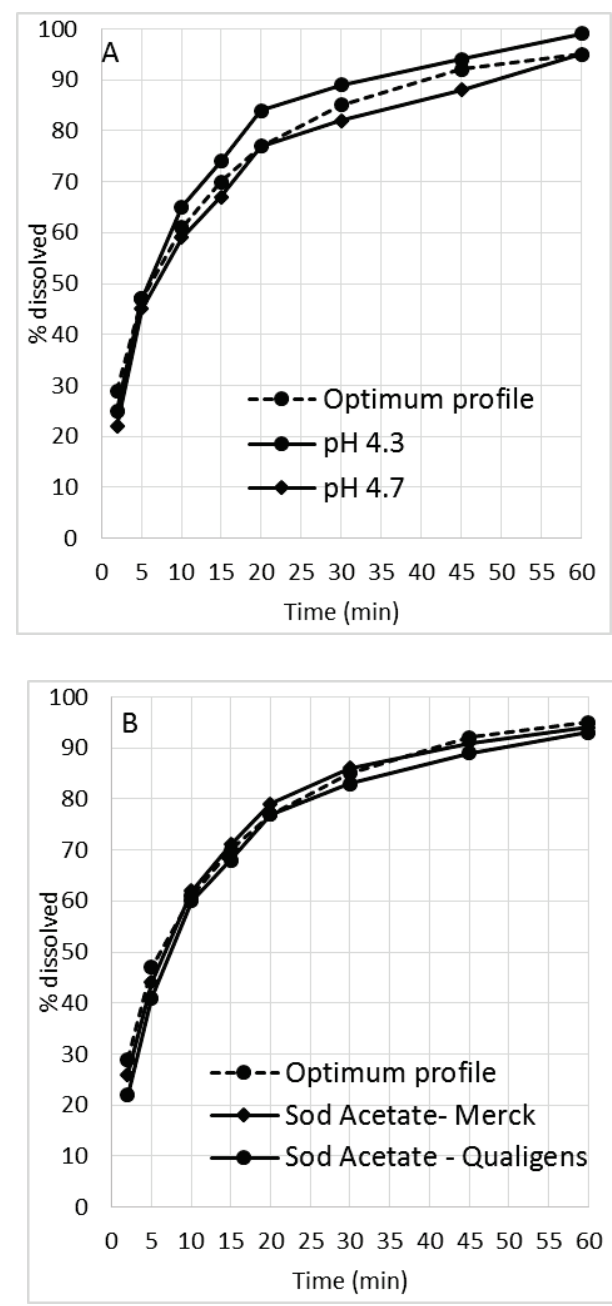

Figure 6. Effect of $(A) p H$ and $(B)$ grade of critical excipients in buffer preparation.

\section{CONCLUSIONS}

The study showcased the systematic and sequential design of experiments in the development of a discriminatory dissolution method for a BCS Class 2 drug exhibiting highly $\mathrm{pH}$-dependent solubility. The parameters for the robust and discriminatory dissolution method for etoricoxib tablets are $900 \mathrm{~mL}$ of $\mathrm{pH} 4.5$ acetate buffer in Apparatus 2 at 100 rpm, which can be conveniently utilized for product quality control. Similar efforts in the development and reporting of dissolution methods benefit the industry at large and government organizations in the critical evaluation of pharmaceutical products.

\section{CONFLICT OF INTEREST}

No conflict of interest has been declared by the authors.

\section{DEDICATION}

Yasvanth Ashokraj dedicates this article to Prof. Ramesh Panchagnula, Vice President and Head, Nektar India.

\section{REFERENCES}

1. Dressman, J. B.; Amidon, G. L.; Reppas, C.; Shah, V. P. Dissolution Testing as a Prognostic Tool for Oral Drug Absorption: Immediate Release Dosage Forms. Pharm. Res. 1998, 15 (1), 11-22. DOI: 10.1023/A:1011984216775.

2. Raju, V.; Murthy, K. V. R. Development and validation of new discriminative dissolution method for carvedilol tablets. Indian J. Pharm. Sci. 2011, 73 (5), 527-536. DOI: 10.4103/0250-474X.99000.

3. Anand, O.; Yu, L. X.; Conner, D. P.; Davit, B. M. Dissolution Testing for Generic Drugs: An FDA Perspective. AAPS J. 2011, 13 (3), 328-335. DOI: 10.1208/s12248-011-9272-y.

4. Darling, D. Regulatory Expectations of Presentation of Dissolution Data. Presented at the 3rd APS International PharmSci Conference, Nottingham, UK, Sept 12-14, 2012. http://www.ukpharmsci. org/2012resourcepack/PDFPresentations/TA_1035_ David_Darling.pdf (accessed Feb 1, 2016).

5. Arcoxia film-coated tablets. electronic Medicines Compendium (eMC) Web site. http://www. medicines.org.uk/emc/medicine/29136 (accessed Feb 1, 2016).

6. Okumu, A.; DiMaso, M.; Löbenberg, R. Computer simulations using GastroPlus ${ }^{\mathrm{TM}}$ to justify a biowaiver for etoricoxib solid oral drug products. Eur. J. Pharm. Biopharm. 2009, 72 (1), 91-98. DOI: 10.1016/j. ejpb.2008.10.019.

7. $<1092>$ The Dissolution Procedure: Development and Validation. In The United States Pharmacopeia and National Formulary USP 36-NF 31; The United States Pharmacopeial Convention, Inc.: Rockville, MD, 2013.

8. Fish, W. P.; Young, J.; Shah, P.; Gao, Z. The Use of Experimental Design Principles in Dissolution Method Development: Development of a Discriminating Dissolution Method for Sprycel Film-Coated Tablets. J. Pharm. Innovation 2009, 4 (4), 165-173. DOI: 10.1007/s12247-009-9071-5.

9. Wu, Y.; Kildsig, D. O.; Ghaly, E.S. Effect of Hydrodynamic Environment on Tablets Dissolution Rate. Pharm. Dev. Technol. 2004, 9 (1), 25-37. DOI: 10.1081/PDT120027415 .

10. Calcium Phosphate, Dibasic Anhydrous. In Handbook of Pharmaceutical Excipients, 6th ed.; Rowe, R. C, Sheskey, P. J., Quinn, M. E., Eds.; Pharmaceutical Press: London, 2009; pp 94-96. 